\title{
Adaptive laboratory evolution - principles and applications for biotechnology
}

\author{
Martin Dragosits ${ }^{{ }^{*}}$ and Diethard Mattanovich ${ }^{2}$
}

\begin{abstract}
Adaptive laboratory evolution is a frequent method in biological studies to gain insights into the basic mechanisms of molecular evolution and adaptive changes that accumulate in microbial populations during long term selection under specified growth conditions. Although regularly performed for more than 25 years, the advent of transcript and cheap next-generation sequencing technologies has resulted in many recent studies, which successfully applied this technique in order to engineer microbial cells for biotechnological applications. Adaptive laboratory evolution has some major benefits as compared with classical genetic engineering but also some inherent limitations. However, recent studies show how some of the limitations may be overcome in order to successfully incorporate adaptive laboratory evolution in microbial cell factory design. Over the last two decades important insights into nutrient and stress metabolism of relevant model species were acquired, whereas some other aspects such as niche-specific differences of non-conventional cell factories are not completely understood. Altogether the current status and its future perspectives highlight the importance and potential of adaptive laboratory evolution as approach in biotechnological engineering.
\end{abstract}

Keywords: Laboratory evolution, Cell factory engineering, Microbial growth, Microbial stress, Biotechnology

Microbial cells are of special importance for biotechnological applications and bacteria and yeasts are used for many biotechnological tasks, ranging from biofuel production [1-3], commodity chemical synthesis [4,5], through to the production of industrial and biopharmaceutical proteins [6-8]. Moreover, biological catalysis offers several advantages over chemical synthesis, such as stereo-selective production of chemical compounds [9]. Synthetic biology is a new emerging field $[10,11]$ and systems metabolic engineering has proven very successful for the design and implementation of biotechnological production processes [12-14]. Recently, classical genetic engineering was successfully complemented with artificial laboratory selection of microbial cells in order to generate potentially robust and optimized microbial production systems $[15,16]$.

Here we summarize the basics and implications of adaptive laboratory evolution for biological engineering. We present important studies on laboratory evolution and evolutionary engineering with the aim of improving microbial growth on relevant substrates and stress

\footnotetext{
* Correspondence: martin.dragosits@boku.ac.at

'Department of Chemistry, University of Natural Resources and Life Sciences, Muthgasse 11, A-1190 Vienna, Austria

Full list of author information is available at the end of the article
}

resistance. Furthermore, we discuss the existence of cross-stress dependencies in bacteria and yeasts and the implications of evolutionary engineering for nonconventional host organisms. It is clear that the vast amount of available data on this topic exceeds the scope of this recapitulation. Towards this end we want to point out that some other recent reviews discussed the importance of adaptive laboratory evolution (ALE) in applied microbiology and may cover other relevant topics [15-19]. Furthermore, additional reviews cover more general aspects of ALE [20-23].

\section{Adaptive laboratory evolution}

Adaptive laboratory evolution as a scientific approach is very important towards the analysis of evolutionary phenomena in a controlled laboratory setting. The principles on which laboratory evolution experiments are based, date back to scientists such as Antonie van Leeuwenhoek, Louis Pasteur, Robert Koch and most notably Charles Darwin, with their discoveries of microorganisms, the general acceptance of the germ theory and the importance of natural and artificial selection for biological evolution and breeding. Hence, adaptive laboratory evolution was already performed about a hundred years ago by William Dallinger [22] and during the middle of the last century
Ciomed Central

(c) 2013 Dragosits and Mattanovich; licensee BioMed Central Ltd. This is an Open Access article distributed under the terms of the Creative Commons Attribution License (http://creativecommons.org/licenses/by/2.0), which permits unrestricted use, distribution, and reproduction in any medium, provided the original work is properly cited. 
$[24,25]$; however, particularly in the last 25 years, there has been an ever increasing number of such experiments with Escherichia coli and Saccharomyces cerevisiae being the most prominent organisms under investigation [26-28].

During microbial ALE, a microorganism is cultivated under clearly defined conditions for prolonged periods of time, in the range of weeks to years, which allows the selection of improved phenotypes. Microbial cells offer important advantages for ALE studies: (a) most microbial cells have simple nutrient requirements, (b) they can be easily cultivated in the laboratory and (c) microbial cells generally grow very fast and can be cultivated for several hundred generations within several weeks or months (with typical specific growth rates of microbial cells in the range of $\mu=0.05$ to $1.0 \mathrm{~h}^{-1}$ ). In contrast to comparative genomics [29], ALE allows phenotypic changes to be clearly associated with a certain growth environment that leads to the selection of traits. Moreover, due to rather new technologies, including transcriptional profiling [30] and massive next-generation DNA sequencing (NGS) [31,32], phenotype-genotype correlations can be easily obtained by whole genome resequencing (WGS). ALE led to important insights and experimental proof for evolutionary biology. On the forefront of laboratory evolution experiments, the long term study of Professor Lenski and his research group at the Michigan State University has to be mentioned. This single parallel $E$. coli adaptation experiment is already exceeding 50000 generations [33-35]. Together with other similar experiments, the scientific community was provided with insights into the genetic basis of increased fitness [36], implications of historical contingency in laboratory evolution [37], second order effects during evolution [38], the interrelation of population size, robustness and evolvability [39-41], clonal interference [42] and evolutionary bet hedging [43].

\section{Laboratory selection methods}

Generally, ALE experiments with microorganisms are easy to establish and the common methods, which are used, are shown in Figure 1a and Figure 1b. Batch cultivation in shake flasks can be performed to propagate microbial cells in parallel serial cultures. At regular intervals (usually on a daily basis), an aliquot of the culture is transferred to a new flask with fresh medium for an additional round of growth. Clearly, this easy setup has the advantages of cheap equipment and the ease of massive parallel cultures. By replacing shake flasks with deep well plates with even smaller culture volumes, hundreds of microbial cultures can be grown in parallel [44]. Several environmental factors can be easily controlled, including temperature and spatial culture homogeneity

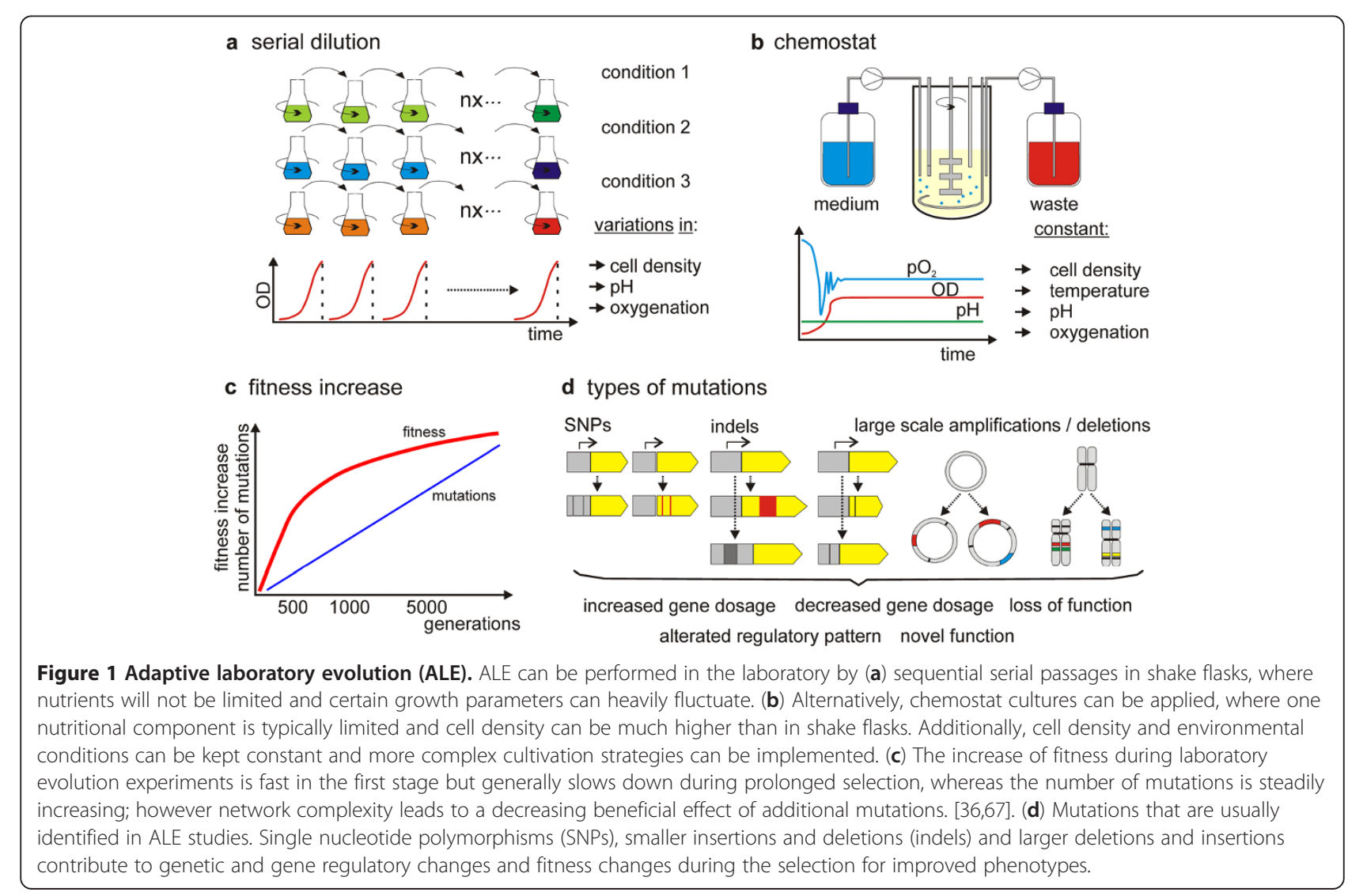


(by constant mixing of the culture). Nevertheless, batch cultivation has certain shortcomings, including varying population density, fluctuating growth rate, nutrient supply and fluctuating environmental conditions such as environmental $\mathrm{pH}$ and dissolved oxygen (partial oxygen pressure, $\mathrm{pO} 2$ ). In many cases, these factors may not be of great importance for the experimental setup but the simplicity of the setup can prevent the implementation of complex environments for microbial selection experiments. As an alternative to serial batch growth, continuous (chemostat) cultures in bioreactor vessels (Figure 1b) are applied [45-50]. The advantages of chemostat cultivation are constant growth rates and population densities. Furthermore, it is possible to tightly control nutrient supply and environmental conditions such as $\mathrm{pH}$ and oxygenation. A major drawback is the costs of operation. Even with small vessels and parallel operation, as they are available from many manufacturers, the costs are by far exceeding the costs of shake flask cultivations. A major difference between prolonged selective growth in batch and chemostat cultures is, however, the growth in nutrient-sufficient versus potentially nutrient-limited conditions. Principally, feedback control mechanisms, such as in the case of a pH-auxostat [51] allow nutrient-sufficient growth in chemostat cultures at the maximum specific growth rate; however, nutrient-limited growth at a growth rate below the maximum is predominantly applied in continuous cultures. As indicated in Figure 1a, in serial batch cultures for ALE, the transfer often occurs before the stationary phase is reached. Clearly, this is a prerequisite to avoid stationary phase adaptation [52]. Thus, microbial cells are predominantly grown in exponential cultures. In contrast, in continuous cultures, the growth rate is kept constant (or in certain experimental setups stepwise / continuously increasing) by the limitation of a major growth nutrient, such as glucose, nitrogen or phosphate. In this context, cells selected under growth limiting conditions can show growth trade-offs in non-limiting conditions and vice versa [53]. If the selection aims at phenotypes that do not rely on increased growth rates or biomass yield but other traits such as e.g. a yes / no survival phenotype after gene knockout or antibiotic production, solid media or combinations of solid and liquid media can be applied in order to select for proper phenotypes $[54,55]$.

There are several critical issues that may arise during the genetic engineering and artificial selection for microbial biotechnology [16]. As example, for certain processes selection for increased tolerance to an accumulating but toxic bio-product can be appropriate. Other biotechnological processes, such as recombinant protein production and metabolite co-production in microbial cells, are coupled to microbial growth [56]. Furthermore, scale-up of microbial processes often represents a major obstacle in process design. Whereas small-scale cultivations and initial engineering steps are often performed in complex media in batch culture, during the scale-up of the process, cells are exposed to altered environmental conditions and environmental stress regimes $[57,58]$.

Altogether, these factors should ideally also be wellconsidered in the case that ALE is implemented in microbial cell factory design. The initial choice of conditions, including media composition and limited versus non-limited growth rate, will greatly influence whether the artificially selected microbial host will be suitable for its dedicated application.

\section{Increased growth and fitness as desirable criteria}

During adaptive evolution, certain trait values change and are associated with increased (Darwinian) fitness [20]; as such, an improved phenotype or property is often equal to increased fitness. During direct competition of an ancestral microbial strain and an adapted strain, the increased fitness of the adapted variant will be obvious by its increased frequency in the total population. With suitable neutral genetic markers, this fitness difference can be easily monitored during laboratory evolution of microbial cells [34,59]. Competitive fitness assays usually involve growth in batch cultures and are balanced for all growth phases (lag, exponential and stationary phase). On the other hand, the maximum growth rate $\left(\mu_{\max }\right)$ of microbial cells is less commonly used in order to determine changes of the evolutionary fitness. Nevertheless, for biotechnology, parameters such as $\mu_{\max }$, survival rates in toxic concentrations of certain chemical compounds and absolute biomass yield are appropriate fitness criteria.

An important factor during the search for improved phenotypes by ALE is the time span for the selection experiment. In summary Tables 1 and Table 2 , it can be seen that a typical ALE experiment is performed for somewhat between 100 and 2000 generations and usually takes a few weeks up to a few months. During ALE, several phenotypes will occur at first and compete for 'dominance' in the total population. Stable phenotypes will accumulate rapidly, although clonal interference [42], bet hedging [43,60], genetic hitchhiking [61] and fluctuating growth environments can lead to significant population heterogeneity $[22,62,63]$. Thus, it cannot be assumed that a homogenous population is present during any point of a laboratory evolution experiment. It should be noted that selection for improved fitness in a specialized environment often leads to significant tradeoffs in other stressful or selective conditions [19]. In this context, for biotechnological purposes, the best phenotype is not necessarily the one with the highest fitness in a certain condition, but the one that shows increased performance and the least trade-offs in other environmental conditions. 
Table 1 Adaptive laboratory evolution experiments with bacteria

\begin{tabular}{|c|c|c|c|}
\hline Species, Strain & Environment & Selection time & Reference \\
\hline \multicolumn{4}{|c|}{ Nutrient } \\
\hline E. coli REL606 & DM minimal medium, glucose & $>50000$ generations & Lenski et al. $[34,36,64]$ \\
\hline E. coli MG1655 & glycerol-limited chemostat & 217 generations & Weikert et al. [50] \\
\hline E. coli & glucose-limited chemostat & 280 generations & Notley-McRobb and Ferenci [49] \\
\hline E. coli MG1655 & M9 minimal medium, glycerol & 700 generations & Ibarra et al. [65] \\
\hline E. coli MG1655 & minimal medium lactate or glycerol & $>1000$ and $>600$ generations & Fong et al. [66] \\
\hline E. coli MG1655 & M9 minimal medium, L-lactate & 900 generations & Hua et al. [67] \\
\hline E. coli MG1655 & M9 minimal medium, lactose & 500 generations & Dekel and Alon [68] \\
\hline E. coli MG1655 & glucose minimal medium & 25 days & Conrad et al. [69] \\
\hline E. coli MG1655 $\Delta p g i$ & M9 minimal medium, glucose & 50 days & Charusanti et al. [70] \\
\hline E. coli MG1655 & M9 minimal glycerol + 1,2 propanediol & 700 generation & Lee et al. [71] \\
\hline \multirow[t]{2}{*}{ E. coli } & chemostat minimal medium & 37 days & Wang et al. [46] \\
\hline & phosphate limitation & & \\
\hline T. fusca & Hagerdhal medium, glucose, cellobiose & 220-284 generations & Deng and Fong [72] \\
\hline L. lactis KF147 & milk & 1000 generations & Bachmann et al. [73] \\
\hline G. sulfurreducens & lactate & nd & Summers et al. [74] \\
\hline C. tyrobutyricum ATCC 25755 & repeated batch fermentations, glucose & 130 days & Jiang et al. [75] \\
\hline E. coli REL606 & DM minimal medium, lactose & 2000 generations & Quan et al. [76] \\
\hline E. coli $B$ & LB xylose, lactate production & 3 month & Zhao et al. [77] \\
\hline \multicolumn{4}{|c|}{ Environmental stress } \\
\hline E. coli & high temperature $\left(41.5^{\circ} \mathrm{C}\right)$ & 2000 generations & Rhiele et al. [78] \\
\hline E. coli & UV light & 80 UV light cycles & Alcantara-Diaz et al. [79] \\
\hline E.coli & Freeze-thaw & 150 cycles & Sleight and Lenski $[80,81]$ \\
\hline E. coli $\Delta r p o s$ & osmotic stress & nd & Stoebel et al. [82] \\
\hline E. coli & LB medium heat stress $48.5^{\circ} \mathrm{C}$ & 620 generations & Rudolph et al. [83] \\
\hline E. coli MG1655 & rich medium , $7 \%(\mathrm{v} / \mathrm{v})$ ethanol & $30-160$ generations & Goodarzi et al. [84] \\
\hline E. coli W3110 & M9 minimal medium, $\mathbf{5 \%}(\mathbf{v} / \mathbf{v})$ ethanol & 1000 generations & Horinouchi et al. [85] \\
\hline E.coli & LB medium $4-8 \mathrm{~g} \mathrm{~L}^{-1}$ isobutanol & 45 transfers & Atsumi et al. [86] \\
\hline E. coli EcNR1 & M9 minimal medium, $\mathbf{0 . 7 5} \%(\mathbf{v} / \mathbf{v})$ isobutanol & 500 generations & Minty et al. [87] \\
\hline Bacillus boroniphilus & TSB medium, $0.055-0.3 \mathrm{M} \mathrm{H}_{3} \mathrm{BO}_{3}$ & 50 transfers & Sen et al. [88] \\
\hline E.coli B & Davis minimal medium, $42.2^{\circ} \mathrm{C}$ & 2000 generations & Tenaillon et al. [89] \\
\hline E. coli MG1655 & LB medium temperature stress (up to $48^{\circ} \mathrm{C}$ ) & 8 months & Blaby et al. [90] \\
\hline E. coli $\mathrm{K}-12$ & M9 medium, glucose,chemostat, $\boldsymbol{n}$-butanol & $>200$ generations & Reyes et al. [91] \\
\hline E. coli MG1655 & $\begin{array}{l}\text { M9 minimal medium, low } \mathbf{p H}, \boldsymbol{n} \text {-butanol, } \mathbf{H}_{\mathbf{2}} \mathbf{O}_{2} \text {, } \\
\text { high salt }\end{array}$ & 500 generations & Dragosits et al. [63] \\
\hline \multicolumn{4}{|c|}{ Miscellaneous } \\
\hline S. clavuligerus 27064 & TSB + MRSA N315 & nd & Charusanti et al. [55] \\
\hline G. oxydans DSM2343 & glycerol, increased DHA production & 25-50 transfers & Lu et al. [92] \\
\hline
\end{tabular}

Experiments are presented in the approximate chronological order for the different selection conditions. The respective selection condition in the second column (environment) is highlighted in bold letters. nd - no data.

Besides the time needed to accumulate improved phenotypes, the degree of increased fitness that can be achieved, is an important factor for efficient implementation of ALE in microbial strain engineering. Based on previous studies, we can estimate that within 100 to 500 generations (corresponding to up to 2 months of selection for a typical E. coli or S. cerevisiae culture), a fitness increase of up to $50-100 \%$ can be achieved. In certain cases ALE can lead to an even higher increase of up to $1000 \%$ as recently shown for the iron reduction rate of 
Table 2 Adaptive laboratory evolution experiments with yeasts

\begin{tabular}{|c|c|c|c|}
\hline Species, Strain & Environment & Selection time & Reference \\
\hline \multicolumn{4}{|c|}{ Nutrient } \\
\hline S. cerevisiae & glucose-limited chemostat & 250 generations & Ferea et al. 1999 [93] \\
\hline S. cerevisiae & glucose-limited chemostat & 500 generations & Dunham et al. 2002 [94] \\
\hline $\begin{array}{l}\text { S. cerevisiae capable of growth } \\
\text { on xylose, EMS-mutagenized, }\end{array}$ & $\begin{array}{l}\text { chemostat, minimal medium, } \\
\text { anaerobic growth on xylose }\end{array}$ & 170 generations & Sonderegger and Sauer 2003 [95] \\
\hline S.cerevisiae & maltose-limited chemostat & $>25$ generations & Jansen et al. 2004 [96] \\
\hline S. cerevisiae CEN.PK $\triangle P D C 1,5,6$ & $\begin{array}{l}\text { chemstat, shake flask, synthetic medium, } \\
\text { C2-independence }\end{array}$ & nd & van Maris et al. [97] \\
\hline S.cerevisiae & glucose-limited chemostat & 200 generations & Jansen et al. 2005 [98] \\
\hline S. cerevisiae RWB 217 & chemostat, batch, xylose & $\mathrm{Nd}$ & Kuyper et al. 2005 [99] \\
\hline S. cerevisiae & synthetic medium, arabinose & 17 transfers, ca. 3500 hours & Wisselink et al. $2007[100,101]$ \\
\hline \multirow[t]{2}{*}{ S. cerevisiae } & chemostat, sequential batch & 40 days and 20 cycles & Wisselink et al. 2009 [102] \\
\hline & glucose, xylose and arabinose & & \\
\hline S. cerevisiae DBY11331 & sulfate-limited chemostat & 188 generations & Araya et al. 2010 [103] \\
\hline S. cerevisiae TMB3061 & $\begin{array}{l}\text { chemostat, synthetic medium, growth } \\
\text { on xylose and arabinose }\end{array}$ & 20 and 65 generations & Garcia Sanchez et al. [104] \\
\hline S. cerevisiae CEN.JB27 $\triangle P Y C 1$ & $\begin{array}{l}\text { Synthetic medium, selection for growth } \\
\text { on glucose }\end{array}$ & nd & Zelle et al. 2010 [105] \\
\hline S. cerevisiae CEN-PK & galactose minimal medium & 400 generations & Hong et al. 2011 [106] \\
\hline $\begin{array}{l}\text { S. cerevisiae, engineered, } \\
\text { scfa }^{+}, \mathrm{Pyc}^{-}\end{array}$ & $\begin{array}{l}\text { batch and nitrogen limited chemostat, } \\
\text { anaerobic glucose }\end{array}$ & approx. 30 days & Zelle et al. 2011 [107] \\
\hline S. cerevisiae & glucose limitation & approximately 100 generations & Wenger et al. 2011 [53] \\
\hline \multicolumn{4}{|l|}{ SC288 } \\
\hline S. cerevisiae & increased xylose fermentation & nd & Shen et al. 2012 [108] \\
\hline S. cerevisiae, engineered & $\begin{array}{l}\text { batch and chemostat cultures, xylose } \\
\text { utilization and ethanol production }\end{array}$ & $70+120$ generations & Zhou et al. 2012 [109] \\
\hline S. cerevisiae CMB.GS001 & Increased aerobic growth on xylose & 10 cycles & Scalcinati et al. 2012 [110] \\
\hline S. cerevisiae $\Delta j e n 1$ & synthetic medium, lactate & 10 tranfers & de Kok et al. 2012 [111] \\
\hline S. cerevisiae & $\begin{array}{l}\text { VERT, YNB, lignocellulosic hydrolysate } \\
\text { tolerance }\end{array}$ & 463 generations & Almario et al. 2013 [112] \\
\hline \multicolumn{4}{|c|}{ Environmental stress } \\
\hline $\begin{array}{l}\text { S. cerevisiae CEN-PK } \\
\text { EMS mutagenized }\end{array}$ & $\begin{array}{l}\text { chemostat and batch selection, } \\
\text { multiple abiotic stresses }\end{array}$ & up to 68 generations & Cakar et al. 2005 [113] \\
\hline $\begin{array}{l}\text { S. cerevisiae CEN-PK } \\
\text { EMS mutagenized }\end{array}$ & $\begin{array}{l}\text { YMM, continuous and pulsed } \mathrm{CoCl}_{2} \\
\text { stress }\end{array}$ & 25 transfers & Cakar et al. 2009 [114] \\
\hline C. albicans & fluconazole & 330 generations & Selmecki et al. 2009 [115] \\
\hline S. cerevisiae BY4741 & YP galactose medium, $0.5 \mathrm{M} \mathrm{NaCl}$ & 300 generations & Dhar et al. 2011 [116] \\
\hline S. cerevisiae BL7 & YP medium, 0 - $2.5 \mathrm{~g} \mathrm{~L}^{-1} \mathrm{CuSO}_{4}$ & nd & Adamo et al. 2012 [117] \\
\hline S. cerevisiae & SD medium, $1.17 \% \mathrm{NaCl}, 37^{\circ} \mathrm{C}$ & 25 generations & Gray and Goddard 2012 [118] \\
\hline S. cerevisiae & YP medium, 6 - $\mathbf{8 \%}$ ethanol & 141 generations & Avrahami-Moyal et al. 2012 [119] \\
\hline \multicolumn{4}{|l|}{ W303 } \\
\hline S. cerevisiae & salt and oxidative stress & 300 generations & Dhar et al. 2013 [120] \\
\hline \multicolumn{4}{|c|}{ Miscellaneous } \\
\hline S. cerevisiae $\Delta$ myo 1 & cytokinesis stress & nd & Rancati et al. 2008 [54] \\
\hline \multirow[t]{2}{*}{ S.cerevisiae EC1118 } & SD gluconate, & 240 generations & Cadière et al. 2011, 2012 [121,122] \\
\hline & enological properties & & \\
\hline
\end{tabular}

Experiments are presented in the approximate chronological order for the different selection conditions. The respective selection condition in the second column (environment) is highlighted in bold letters. nd - no data. 
Geobacter sulfurreducens [123], although this increase was only achieved after 24 months of selection (a time scale that may not be feasible for many biotechnological purposes). The maximum achievable fitness increase within a certain time or generation span can vary significantly depending on the selection pressure. Whereas certain environments can lead to fast selection of improved phenotypes, others, including the long-term adaptation to environmental $\mathrm{pH}[59,63]$, represent complex environments where microbial cells show low adaptive potential in terms of absolute fitness gain. In this context, we cannot clearly predict the scale at which improved phenotypes emerge during laboratory evolution, although recent literature indicates that such predictions are possible in certain growth contexts such as carbon source adaptation [65]. Moreover, it is important to note that the fitness increase as a function of the total number of generations is not linear (Figure 1c). Whereas the fitness increase is usually fast within the first 100 to 500 generations, it slows considerably down during the course of ALE [36]. For standard ALE in biotechnology this is a critical factor, as prolonged selection that exceeds the first rapid evolutionary adaptation phase will not necessarily lead to significantly improved phenotypes [67]; therefore, there is a clear cost benefit ratio of experimental selection time and achievable fitness improvements for most microbial engineering purposes. Thus, complex environments may depend on advanced selection strategies to compensate a low adaptive potential and the ceasing fitness gain during ALE.

Many typical ALE experiments were performed in shake flasks and similar growth conditions with cell densities in the range of $10^{7}$ to $10^{9}$ cells per $\mathrm{mL}$ for typical microbial cultures $[124,125]$ and as such, the total number of generations is commonly used to estimate the emergence of adaptive mutations. Nevertheless, this correlation does not take into account the total number of cells in a population. Only recently, the cumulative number of cell divisions (CCD) was proposed as a more appropriate denominator to evaluate microbial evolution [126].

\section{Mutations and robustness}

Mutations are the basis underlying genetic change and the selection of improved phenotypes in nature as well as in the laboratory. Although DNA replication itself is a process of high fidelity with a mutation rate of approximately $10^{-10}$ per base pair per replication for microbial cells [127], the large population sizes in microbial cultures still allow the emergence of frequent mutations within a short time span.

In recent ALE experiments various types of mutations were detected: Single-nucleotide polymorphisms (SNPs), small scale insertions and deletions (indels), transposable element (insertion sequence, IS) movements and amplifications as well as deletions of larger genomic regions (Figure 1d). Altogether, these genomic changes contribute to the change of trait values during laboratory evolution. As summarized in a recent review, SNPs (61\%, with G:C to T:A mutations being disproportionally frequent) account for the majority of observed genetic changes [15]. Deletions accounted for approximately $29 \%$ of the observed genomic changes, followed by insertions (7\%) and IS movements (3\%) [15]. Similar to SNPs, these types of mutations also contribute to increased and decreased gene dosage, altered or diminished gene function or altered gene regulatory patterns. In several recent studies, gene amplifications were identified to contribute to increased gene dosage $[63,116,128]$ and subsequently increased fitness. In a purely evolutionary perspective, they are even more important. Whereas there is an ongoing debate about the long-term fixation of gene duplications, either by their selective advantage under selection pressure, or by selective neutrality and fixation by random drift, they are a prerequisite for the development of novel molecular functions [129-131].

For microbial, more specifically prokaryotic cells, a new model for the emergence of novel metabolic pathways was introduced. This so-called toolbox model suggests that prokaryotic metabolic pathways emerge through horizontal gene transfer (HGT) and are rapidly acquired and lost based on nutrient availability [132]. Nevertheless, the role of HGT in natural evolution remains a controversial topic [133].

Microbial metabolic networks often provide appropriate functions at a minimal size [134] and furthermore, their complexity seems to be correlated with environmental factors [135]. If metabolic networks operate at a minimal size, the mutational robustness has to be high in order to maintain proper functions. Although counterintuitive at first, it was shown that mutational robustness can have a positive effect on evolvability, depending on the population size and network architecture $[41,136]$. In laboratory evolution experiments, chemical mutagens, mutants deficient in DNA repair or transposon libraries can be applied in order to speed up the selection process by increasing the diversity of targets for selection. Random mutagenesis is also a very popular mechanism in order to select for improved phenotypes in biotechnology $[137,138]$. Interestingly, it was shown that high mutation rates and mutator strains can emerge naturally during laboratory evolution and contribute to the formation of genetic novelty [33,73,139-141]. It is clear that a higher mutation rate can only be beneficial to a certain extent as it also results in a genetic load [142]. Recent data indicated that long-term adaptation, mediated by an increased mutation rate and genetic load, is tightly balanced in bacterial cultures. For 
example, in a recent $E$. coli study, a mutator phenotype mut $T$ was invaded by mut $Y$ mutations. The authors estimated that the mutT mutation leads to an approximately 150 -fold increase of the mutation rate, whereas the mut $Y$ mutations decreased the mutation rate by $40-60 \%$ in order to decrease the genetic load [143]. Furthermore, it was shown that mutational robustness tends to increase with mutation rate and decline with population size [40].

Considering this general tension between mutational robustness of genes and networks and the potential to evolve, enzyme promiscuity is also important for the evolution of novel functions [144]. Moreover, there is evidence that the interplay of protein mutational robustness, protein folding and environmental stress is an additional key factor that determines the evolution of new traits $[145,146]$. The evolution of novel functions and pathways can be hardly observed in a laboratory evolution experiment, even with microbial cells and their fast growth rates. Nevertheless, there are a few examples that highlight that a novel trait can emerge rapidly on a laboratory time scale. For example, an experimental E. coli culture could evolve the ability to aerobically utilize citrate [37], or the ability to use the non-natural carbon source 1,2-propanediol [71]; also, a strain deficient in glutathione biosynthesis was able to develop compensatory mutations to restore this function [147].

Microbial cells are generally grown at constant growth rate in chemostats or at constant $\mu_{\max }$ during the exponential phase of a batch culture. It should be noted that microbial batch cultures can show rapid evolutionary adaptation if they are exposed to prolonged stationary phase. In fact, this nutrient limited condition represents a very dynamic environment for microbial populations. So-called GASP (growth advantage in stationary phase) mutants are rapidly fixed in E. coli populations [52]. Mutations in amino acid catabolic processes and stress response sigma factor, rpoS, are of special importance for acquiring a selective advantage in such nutrient starved conditions [148,149]; however, such mutational adaptations might be counterproductive for some applications of microbial cells in biotechnology.

\section{Adaptation towards nutrient sources and environmental stresses}

In nature as well as in biotechnological processes, microorganisms are challenged with changing environmental conditions, where they experience fluctuations in temperature, $\mathrm{pH}$, oxygenation, atmospheric or hydrostatic pressure but also water and nutrient availability; thus, the selective pressures can be divided in two main categories: nutrient availability and environmental stress [17]. Microbial cells evolved mechanisms to successfully cope with such stressful conditions in order to maintain cellular homeostasis. In contrast to natural environments, in industrial processes microbial cells may be challenged with non-natural stresses that may result in poor and non-competitive process performance. Many ALE studies analyzed the impact and molecular adaptation towards both stresses (Table 1 and Table 2), leading to major findings regarding the emergence of specialists, generalists, the underlying mutations and the applicability of evolved cells for industrial microbiology.

\section{Nutrient stress}

Efficiency of nutrient source utilization is an important aspect of microbial growth and for industrial purposes it may be governed by factors such as substrate cost or increased bioconversion rates. Multiple studies analyzed the adaptive changes in microbial populations upon nutrient selection pressure.

In a 1997 study, E. coli was evolved in chemostat cultures in order to select for improved growth on glycerol. The authors reported phenotypic changes in terms of colony morphology accompanied with increased growth rate and biomass yield and decreased acetate formation [50]. Subsequently, it was shown that suboptimal growth on glycerol can approach its theoretical maximum during laboratory evolution [65]. In a more recent study, rpoC RNA polymerase (RNAP) mutations were found to be a major source for improved growth on glycerol during batch selection [69]. In this study, the biomass yield increased up to $40 \%$ and acetate overflow was reduced, whereas the metabolic rate increased and the evolved cells showed a lowered or total loss of motility. The rpoC mutations that were identified led to altered gene expression patterns by a global redistribution of transcriptional units (TUs) from ribosomal RNAs to other units.

Other studies focused on the adaptation of E. coli to growth on glucose. In this context the Lenski long-term experiment with 12 replicate cultures is performed with glucose as carbon source. During the first few thousand generations, glucose-evolved cultures developed larger cell size and a lower final cell density as compared with the ancestor [34]. Also, a significant parallelism in the change of the gene expression profiles was noticed in these parallel cultures [64]; WGS of the parallel populations revealed that most cultures acquired mutations in the transcriptional regulator $n a d R$, the pyruvate kinase pykF, the rbs operon, the transcriptional regulator malT and the stringent response regulator spoT [36]. Furthermore, changes in DNA topology contributed to global transcriptional changes by altered DNA superhelicity, mediated by mutations found in genes such as fis, top $A B, \operatorname{gyr} A B$ and $d u s B$ [150]. In contrast to batch selected populations, during aerobic glucose-limited chemostat growth in parallel cultures for 280 generations, mutations in the glycoporin $\operatorname{lam} B$ and the 
regulator malT were identified as the cause of long-term adaptation. Moreover, within a single chemostat culture, the authors observed multiple stably co-existing malT mutations [49]. Strikingly, the authors also noticed that malT regulatory mutations, although common in aerobic conditions, were absent during oxygen-limited chemostat growth, where mutations in the glucose permease pts $G$ were common [48]. In another study, the adaptation of a phosphoglucose isomerase ( $p g i)$ deficient strain to glucose was analyzed [70]. Growth rates and glucose uptake rates increased up to 3.6 and up to 2.6 fold in evolved $\Delta p g i$ strains as compared with the ancestor and were accompanied with different phenotypes regarding acetate and formate production. The authors found frequent point mutations in rpoS, $u d h A$ (soluble transhydrogenase), pntAB and a prophage deletion. They did not observe these mutations in the glucose-adapted wildtype strain; other regulatory mutations (including rpo $A B C$ ) occurred in both backgrounds and thereby show, how changes in the gene regulatory network lead to divergent evolutionary results. Frequent rроB mutations were also found during batch evolution on glucose in a more recent study [63], but most importantly the rpoS mutation alone did not positively influence the growth rate in the ancestral $\Delta p g i$ strain; rpoS mutations were epistatically linked to additional mutations.

For other, tightly regulated carbon sources such as lactose in E. coli, it was shown that lacZ expression levels were evolutionary fine-tuned depending on the lactose concentration in the environment, in order to balance the cost of lac $Z$ expression and increased fitness [68]. Moreover, recent data showed that E. coli can evolve different modes of lac $Z$ expression, depending on the environment that contained either lactose, glucose or both as carbon sources. Mutations that lead to altered expression patterns accumulated predominantly in lacI and lacO1 regions of the lac operon [76]. Altogether, these mutations were responsible for a reduced (diauxic) lag phase and increased maximum growth rates in lactose growth medium.

Growth on other carbon sources such as lactate also leads to altered phenotypes, such as decreased acetate overflow in order to tune the cellular energy metabolism [67]. Phosphate limitation experiments resulted in genotypic and phenotypic divergence of E. coli through mutations in rpoS, spoT and $h f q$, which led to the de-regulation of pho genes and increased phosphate transport [46]. Finally, a recent study applied laboratory evolution in order to allow homofermentative L-lactic acid production from xylose in recombinant E. coli. By serial transfers the authors were able to improve anaerobic growth on xylose, although the genetic basis remains yet to be determined [77].
Similarly to E. coli, nutrient adaptation was also extensively studied in S. cerevisiae. Early studies in glucoselimited chemostats revealed that glucose-limited selection led to increased biomass yield and a decreased fermentative capacity $[93,98]$. Decreased activity levels of enzymes related to glycolysis but also correlating changes in the expression levels of genes such as ENO1, ENO2, TDH1 and PYK1 and down-regulation of the stress response $(M S N 2 / 4)$ were observed. Altered cell morphology was also observed, as well as higher affinity towards glucose, although no expression change in the major HXT low-affinity glucose transporters was observed [98]; another study reported the emergence of aneuploidy during glucose-limited growth and amplification of the HXT6 gene with significant divergence between co-evolved populations [94]. Glucose import, sensing and regulation represent major targets during glucose-limited chemostat growth, as a further study also identified frequent mutations in $H X T$ genes and glucose sensors and regulators such as RGT1 and MIG2 [53]. In another study, van Maris and co-workers evolved a S. cerevisiae strain with deficient pyruvate decarboxylase activity for high-glucose tolerance [97]. Subsequently, it was discovered that this glucose tolerant phenotype was due to an in-frame deletion in the glucose regulator MTH1, which led to altered stability of the MTH1 encoded protein [151]. Similarly, Zelle and co-workers established growth on glucose by genetic- and evolutionary engineering in a pyruvate carboxylase-negative $S$. cerevisiae strain. The authors identified a point mutation in the recombinant $s f c A$ gene, which shifted the cofactor preference from NADH to NADPH [107].

For other carbon sources, it was shown that selection for improved galactose utilization could be achieved by serial transfer with a $24 \%$ increase of $\mu_{\max }$ within 62 days. Galactose uptake rates and ethanol production were increased. Transcript analysis showed up-regulation of trehalose and glycogen metabolism, whereas no mutations were identified in galactose or storage carbohydrate related genes. Interestingly, the authors rather identified mutations in RAS/PKA signaling as a source for increased fitness on galactose medium [106].

In xylose adaptation studies, increased growth rates and higher ethanol yields were observed in recombinant yeast. In this case, trehalose and glycogen metabolic processes were significantly down-regulated, nutrient and stress signaling was affected by down-regulation of YAK1 and also accompanied by down-regulation of CWP1 and consequently major cell wall changes [108]. The authors did not identify expression changes in HXT genes, although other studies on improved xylose and arabinose utilization indicated that $H X T$ genes may also be involved in increased xylose uptake rates [99,104]. Furthermore, experiments on pentose utilization highlighted that 
increased gene copy numbers of the heterologous genes in recombinant S. cerevisiae can emerge during evolutionary engineering and contribute to increased growth in anaerobic conditions $[104,109]$. Finally, another example of nutrient stress selection in S. cerevisiae showed that sulfate limitation led to mutations affecting TOR signaling via an RRN3 mutation and a genomic amplification of the highaffinity sulfate transporter SUL1 [103].

Although rare at this point, at least one evolution experiment showed that the general principles that were observed for E. coli and S. cerevisiae, including the tendency towards optimized biomass yield and overflow metabolism, large-scale regulatory changes and mutator strains also emerge in other microbial hosts, such as Lactococcus lactis [73].

\section{Environmental stress}

Among environmental stresses, adaptation to temperature has been investigated in multiple E. coli studies. Riehle and co-workers reported that heat-inducible genes, including $h s l T, f k p A$ and $g a p A$, showed adaptive expression changes during prolonged exposure to increased temperature [78]. In another study it was found that the adaptation to extreme temperature relied on the constitutive expression of GroEL/ES in E. coli [83], whereas a similar study with a different selection procedure identified mutations in $g l p F$ and $f a b A$, accompanied with increased membrane fatty acid saturation as critical factors towards increased thermo-tolerance [90].

Increased growth during osmotic stress was linked to mutations that led to increased expression of enterobactin biosynthesis genes (fepA and entD among others) [63]. Furthermore, it is known that rpoS plays a crucial role towards survival in high osmotic conditions. It was shown that an E. coli population with a rpoS deletion can still develop increased salt tolerance during laboratory evolution by uncoupling of rpoS dependent expression of the ots $B A$ operon, which is important for trehalose synthesis [82].

Apart from temperature and salt stress adaptation, organic solvent tolerance has been a major research topic in recent years, due to its importance for next generation biofuel production. Adaptive changes towards ethanol tolerance were linked to the up-regulation of oxy $R$ and $n r d R$, indicating major changes in the cellular respiratory system but also changes in amino acid metabolism [85]. Furthermore, changes in enterobactin biosynthetic processes (ent genes) were also observed in independent studies $[84,85,91]$. Increased ethanol tolerance in $E$. coli can also be mediated by increased catabolism of ethanol through the TCA cycle [84]. Three other studies dealt with the adaptive evolution towards increased iso- and $n$-butanol stress $[63,86,87]$. Mutations in $\operatorname{acr} A B$ and marC were found independently in these studies; further changes were linked to carbon and nitrogen metabolism via gat $Y$ and thaA [86], enterobactin synthesis [63] or attenuated $r p o S$ activity via $h f q$ mutations [87]. Moreover, mutations identified in isobutanolresistant strains showed a high degree of epistasis [87]. The occurrence of enterobactin related mutations in multiple ALE studies highlight the importance of tuning the cellular redox machinery during environmental stress exposure of E.coli. Furthermore, increased oxidative stress resistance in $E$. coli may be linked to mutations in the soxR reducing system and increased levels of cellular catalase/ peroxidase (katG) [63].

In the model yeast $S$. cerevisiae, adaptation towards salt tolerance led to increased cell size and increased ploidy. Additionally, gene expression changes in CTT1 and MSN4 were observed, as well as a high-frequency SNP in the transcriptional regulator MOT2 [116]. In another study, increased ethanol tolerance was mediated by mutations in the translational regulator SSD1 and UTH1, a protein of unknown function, and indicated that cell wall stability is a major factor in ethanol tolerance of S. cerevisiae [119]. Increased copper tolerance was reported to be mediated by a genomic amplification of CUP1, decreased basal levels of the copper transporters CTR2 and CCC2 and decreased activity of antioxidant enzymes $[117,128]$. Increased tolerance of $S$. cerevisiae towards lignocellulosic hydrolysates such as acetic acid and furfural was correlated with adaptive changes of the oxidative stress response [112].

Altogether, large scale transcriptional re-arrangements and gene amplifications are common during laboratory evolution. As such, ALE studies highlight that duplications and large scale genomic variations, although they can lead to significant issues such as genomic instability [152], play a major role in e.g. yeast evolution; this is also indicated by data on wine yeasts, galactose utilization, comparative genomics and drug resistance [115,153-156]. From these data it can be concluded that: (a) long-term adaptation leads to both largescale transcriptional de-regulation and optimization of terminal nodes (fine-tuning of the expression levels of individual genes), (b) although parallelism is often observed in replicate cultures or even among different laboratories, different evolutionary paths (divergence) can lead phenotypic convergence and (c) epistasis is a major factor for adaptive optimization of gene regulation [89,157]. Importantly, epistatic interactions can be hardly inferred from metabolic modeling studies although significant epistatic connections for strain engineering may be easily identified in ALE studies.

\section{Evolutionary trade-offs and cross-protection}

Microbial cells have an intrinsic capacity to balance self preservation (stress protection) and nutritional competence (SPANC). This SPANC balance was intensively 
studied in E. coli and is governed by strict control of processes such as stress and stringent response $[158,159]$. Recently it was also shown that yeast has a similar intrinsic sensing mechanism to balance growth and stress resistance [160,161]. Evolutionary studies as described above highlight how transcriptional changes can shift the balance from one to the other in order to shut down costly but gratuitous processes. In this context, transcriptional changes during laboratory evolution and specialization can lead to trade-offs or cross-benefits in alternative environments.

Glycerol-limited growth led to stable mutants in a single E. coli population, where one mutant showed increased growth in glycerol-sufficient batch cultures and a second mutant did not. Furthermore, the mutant that showed increased growth in batch culture also showed increased fitness under general nutrient limitation, heat and osmotic stress [50]. On the other hand, long term batch cultivation on glucose led to a rapid decay of nonused metabolic functions, including growth on D-ribose and L-glutamine, in E. coli [35], whereas other studies reported improved growth on many non-evolutionary carbon sources after batch selection with gluconeogenic carbon sources $[66,67]$.

Similarly, aerobic chemostat-selected S. cerevisiae cells also showed increased fitness in anaerobic glucoselimited chemostats and in acetate-limited chemostats, but decreased fitness in nitrogen-limited batch cultivations [53]. Yeast cells that were evolved for efficient galactose utilization [106] subsequently showed trade-offs when grown on glucose as carbon source. This effect was due to antagonistic pleiotropy that was based on mutations of the regulatory protein RAS2 [162].

Regarding the adaptation towards environmental stresses, it was shown for E. coli that increased ethanol tolerance comes at the cost of decreased resistance to acidic conditions [84] and $n$-butanol tolerance is weakly compatible with oxidative stress [63] and leads to tradeoffs in hexane and chloramphenicol resistance [86]. Furthermore, strains evolved under phosphate limitation showed trade-offs in sodium chloride and oxidative stress survival due to decreased rpoS levels [46].

In contrast, it was shown for low temperature evolved E. coli populations that trade-offs at non-evolutionary high temperatures are not universal as individual lineages showed no trade-offs or even a fitness increase [163]. Similarly, $n$-butanol tolerance was reported to be highly compatible with osmotic stress [63].

Examples for adaptive cross-protection are also available for $S$. cerevisiae where cobalt resistance also improved tolerance for other metal ions and pulsed cobalt stress exposure during laboratory evolution even resulted in evolutionary cross-protection to thermal and oxidative stress [114]. A recent study showed that cross- protection towards lignocellulosic hydrolysates can be evolved in S. cerevisiae. Interestingly, several mutants showed increased fitness in the presence of inhibitor combinations but reduced fitness when exposed to individual hydrolysate components [112]. Another interesting study analyzed to which extent multi-stress resistant S. cerevisiae populations can be evolved. The authors tested various chemostat and batch stress regimes and found that batch selection for freeze-thaw tolerance also selected for increased thermal, oxidative and ethanol stress resistance [113].

Thus, evolutionary trade-offs and cross-protection against process-relevant parameters are ubiquitous, but the mode of cultivation and the specificities of the selection pressure largely determine to which extent these phenomena arise.

\section{Non-conventional hosts - implications of ecological niches and genetic network architecture}

Mesophilic organisms such as E. coli and S. cerevisiae have inherent properties that limit their use in certain applications, e.g. high temperature processes. Therefore, non-conventional microbial species play an important role in biotechnology [164-168].

Currently, limited data on the physiology of such organisms still represent a significant obstacle towards rational host cell engineering. Although there are many similarities in the biochemical networks across most microbial hosts, it is well documented that species and environmental niche-specific differences evolved. Whereas core sub-networks and pathways tend to be well conserved, the up- and downstream connections to other cellular networks vary significantly among different species and lead to species-specific differences of stress resistance in adverse environments [169,170] (Figure 2). The evolutionary mechanisms described in the preceding sections are crucial towards the development of such specializations [130,156,171]. They allow anticipatory and foraging behavior in pro- and eukaryotic microbial cells [172-174]. In fact, a recent study in S. cerevisiae indicated that anticipatory gene regulatory patterns can evolve under cycling salt and oxidative stress conditions on a laboratory time scale within 300 generations and that cross-stress protection against salt and oxidative stress is strikingly asymmetrical, with oxidative stress protecting against salt stress but not vice versa [120]. Another recent study in E. coli showed that, by using increased genotypic diversity leveraged by a transposon library, evolutionarily 'old' anticipatory responses can be rapidly decoupled [172].

Certain functions such as overflow metabolism in $S$. cerevisiae may have evolved in order to provide an evolutionary advantage in specific environments and 


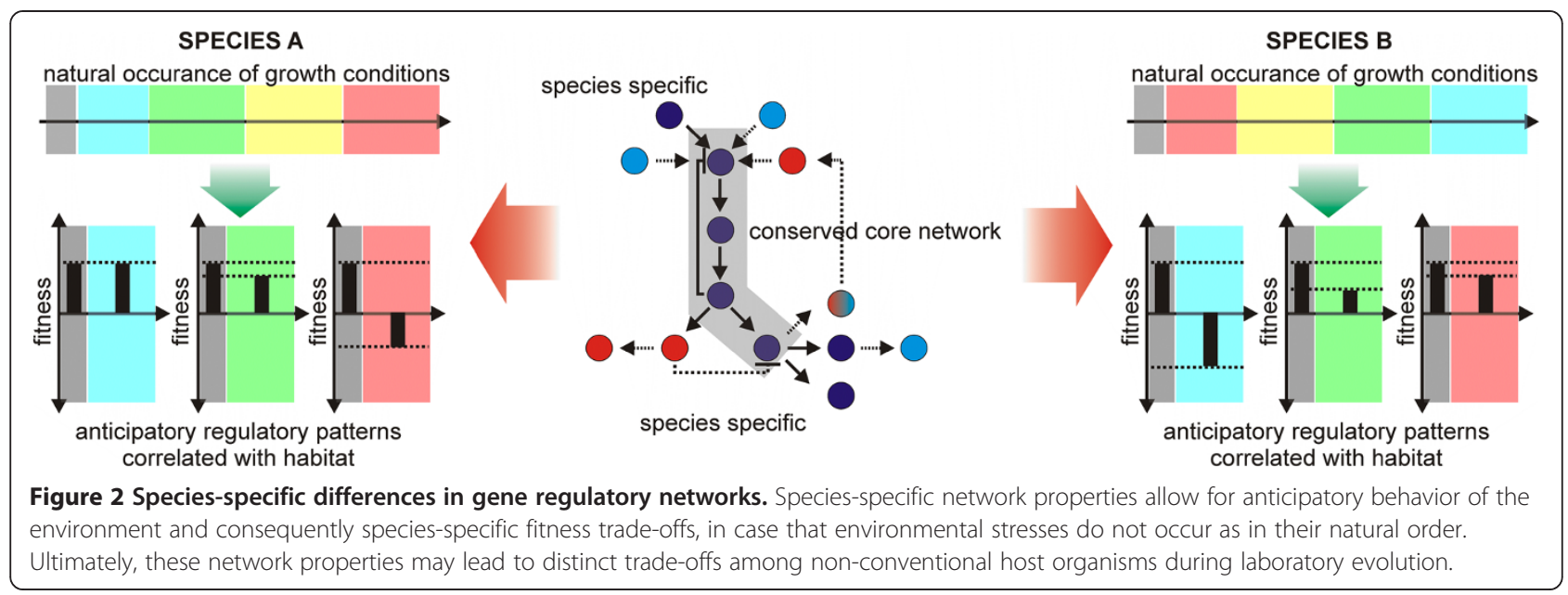

to protect carbon resources from other competing species [175]. Consequently, there are differences in the regulatory blueprints of different species [169,176,177], which can ultimately lead to distinct evolutionary stress tradeoffs during selection. Towards this end, a recent study with S. cerevisiae and Saccharomyces paradoxus showed that the evolutionary rescue (ER) frequency was positively correlated with stress concentration during 100 generations of evolution in S. cerevisiae but negatively correlated in S. paradoxus [44].

It can be concluded that insights into the adaptive cross-stress dependencies of one organism cannot infer the dependencies in a second organism with a distinct evolutionary background. As such, biotechnologists have to be aware that different (evolved) microbial hosts are likely to show distinct trade-offs during process-related stress exposure.

\section{Future directions}

Artificially-evolved microbial cells can be directly used for biotechnological processes (Figure 3). However, based on the data that are available to date, the direct usage comes with certain drawbacks. Biological engineers aim at improving cellular phenotypes by targeted genetic engineering and an evolved microbial strain can impair such engineering approaches because of its unknown genotype, which bears the risk of e.g. hitchhiking of unfavorable mutations that can have adverse effects during environmental stress exposure. In many respects, the same aim can be achieved by ALE and classical genetic engineering. For example, laboratory selection can lead to large-scale rewiring of gene regulatory networks, but an approach, that the authors called gTME, global transcription machinery engineering, can also yield similar results [2]. Nevertheless, with laboratory evolution balanced protein expression levels may be achieved more easily than with standard molecular biology techniques. Moreover, for eukaryotic host organisms such as yeasts, alternating asexual and sexual propagation during selection can be applied in order to decouple beneficial and unsound mutations and thus reduce potential deficits of laboratory evolution experiments [118]. Nevertheless, efficient evolutionary engineering of industrial S. cerevisiae strains has been suggested to be limited by polyploidy, due to increased genetic robustness as compared with haploid laboratory strains [104,178].

In this context, laboratory evolution can be combined with other methods in order to achieve effective biotechnological engineering (Figure 3). Some recent studies highlight the suitability of a highly integrated approach. For example, several studies improved the carbon source utilization of $S$. cerevisiae strains by genetic engineering in combination with evolutionary adaptation [104,107-109]. Trait selection during laboratory evolution can be restricted by the limited number of beneficial mutations that occur on a reasonable time scale. In silico experiments indicate that stepwise evolution can increase microbial evolvability [179]; as such, sequential short rounds of evolution may be used to increase the efficiency of ALE. The genotypic diversity can also be increased to speed up evolution. In a recent study towards increased $n$-butanol tolerance in $E$. coli, a drastic increase in solvent tolerance was rapidly achieved by combining laboratory evolution and genome shuffling of the evolved clones [91]. Other studies used ethylmethanesulfonate (EMS) treated Bacillus boroniphilus to select for increased boron resistance [88] or EMS-treated S. cerevisiae in order to achieve increased nutrient utilization and stress resistance [95,113,114]. Similarly, MAGE (multiplexed automated genome engineering) technology makes use of massively increased genotypic diversity in order to generate improved phenotypes such as improved lycopene production in E. coli and accelerate evolution [180].

Microbial gene regulatory networks are generally very robust towards perturbations and large-scale re-wiring 


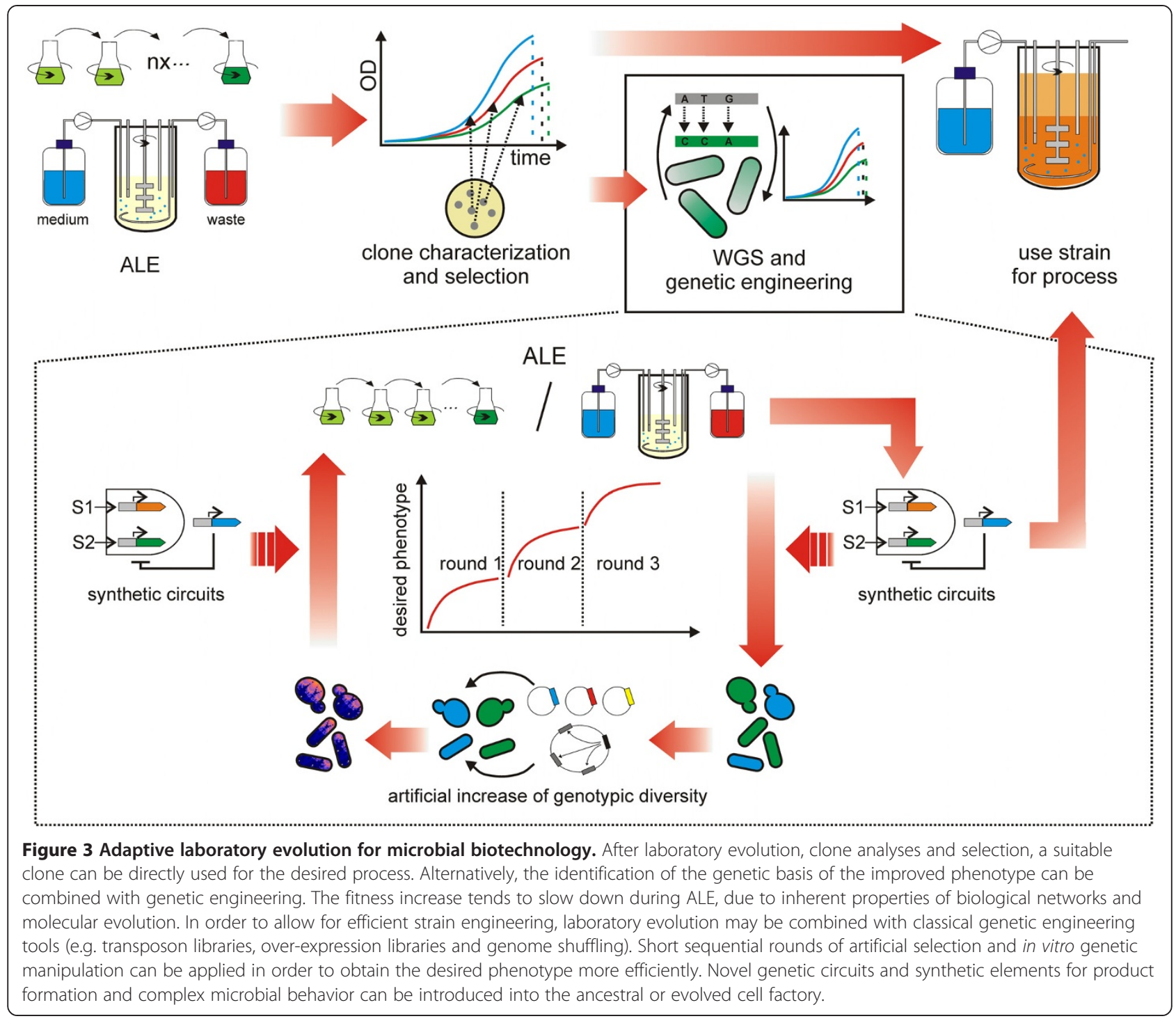

results in a significant number of viable clones with increased evolvability as compared with the wildtype [181]. In this context, a heterologous transcriptional regulator from Deinococcus radiodurans was recently introduced into E. coli in order to select for improved phenotypes [182] and such extensive perturbations of regulatory networks may greatly expand our possibilities in rapid laboratory evolution towards complex traits. Another prospective approach is the co-cultivation of multiple organisms. Besides insights into co-evolutionary behavior, this approach can be exploited in order to increase antibiotic production such as in the case of co-cultivation of Streptomyces clavuligerus and Staphylococcus aureus [55]. Furthermore, in the last decade synthetic biology emerged as a very promising route in biological engineering. In contrast to classical approaches it is highly structured and biological networks are split into smaller functional modules $[11,183]$, leading to complex optimization needs.
Nevertheless, Darwinian selection and adaptive evolution has also been proposed as a potential optimization strategy in this field [184].

Finally, the implications of computational biology for laboratory evolution have to be addressed. Full-scale models of microorganisms are available $[185,186]$ and we can simulate microbial growth. Consequently, genome scale pathway modeling itself proved extremely powerful for metabolic engineering of microbial cells [187]. Similarly, in silico approaches are widely applied in order to address evolutionary questions [40,172]. Computational models are well-suited to study evolutionary change on the population level, whereas in silico evolution of artificial cells that include simulated regulatory networks are currently very limited [21]. Enormous computational power is necessary to simulate living cells, even on a non-evolutionary scale. Additionally, even for well-studied organisms such as E. coli and 
S. cerevisiae, there is still a significant number (approximately $30 \%$ ) of genes with unknown function. Furthermore, the complex interactions of living organisms with their environment are not well understood. Specifically, our understanding of the effect of many environmental stressors on cells, their precise action on certain cellular components, the mode of stress sensing, cellular signal transduction and cross-talk between signal transduction pathways is still limited. These deficits impede the correct implementation of such constraints into computational models. In this context, computational approaches provide important benefits towards rational engineering of microbial cell factories but as it can be foreseen right now, they cannot replace or prevent 'trial and error' laboratory experiments for the foreseeable future.

\section{Conclusions}

As François Jacob pointed out: "Evolution is a tinkerer", and as such evolution is constrained to work on available blueprints. However, genetic engineers have the possibility to rapidly construct novel circuits. The integration of adaptive laboratory evolution into metabolic engineering of microbial cells offers tuning possibilities at multiple levels of the engineering process. Towards this end, future biological engineering studies will also greatly benefit from the insights into microbial physiology that were already acquired by adaptive evolution experiments. Nevertheless, it seems necessary to expand such studies to non-conventional organisms. By combining two worlds, where we rely on the innovative power of the human mind and nature's inherent ability to optimize existing building blocks in a non-directional manner under selective pressure, significant advances in microbial cell factory design can be expected.

\footnotetext{
Abbreviations

ALE: Adaptive laboratory evolution; CCD: Cumulative number of cell divisions; DHA: Dihydroxyacetone; EMS: Ethylmethanesulfonate;

ER: Evolutionary rescue; GASP: Growth advantage in stationary phase; GSR: General stress response; gTME: Global transcription machinery engineering; HGT: Horizontal gene transfer; indel: Insertion / deletion; IS: Insertion sequence; MAGE: Multiplex automated genome engineering; NGS: Next generation sequencing; RNAP: RNA polymerase; rRNA: Ribosomal RNA; SNP: Single nucleotide polymorphism; SPANC: Self preservation and nutritional competence; TU: Transcriptional unit; VERT: Visualizing Evolution in real-time; WGS: Whole genome sequencing.
}

\section{Competing interests}

The authors declare that they have no competing interests.

\section{Authors' contributions}

MD and DM wrote the manuscript. Both authors read and approved the final manuscript.

\section{Acknowledgements}

We would like to thank lain B. H. Wilson and Julia Krasensky for their useful comments on the manuscript. Furthermore, we appreciate the valuable input of the anonymous reviewers, which helped us to improve the quality of the manuscript. Special thanks to the University of Natural Resources and Life Sciences Vienna for financially supporting the publication of this review.

\section{Author details}

${ }^{1}$ Department of Chemistry, University of Natural Resources and Life Sciences, Muthgasse 11, A-1190 Vienna, Austria. ²Department of Biotechnology, University of Natural Resources and Life Sciences, Vienna, Austria.

Received: 22 April 2013 Accepted: 24 June 2013

Published: 1 July 2013

\section{References}

1. Clomburg JM, Gonzalez R: Biofuel production in Escherichia coli: the role of metabolic engineering and synthetic biology. Appl Microbiol Biotechnol 2010, 86:419-434.

2. Alper H, Moxley J, Nevoigt E, Fink G, Stephanopoulos G: Engineering yeast transcription machinery for improved ethanol tolerance and production. Science 2006, 314:1565-1568

3. Kalia VC, Purohit HJ: Microbial diversity and genomics in aid of bioenergy. $J$ Ind Microbiol Biotechnol 2008, 35:403-419.

4. Lee JW, Kim HU, Choi S, Yi J, Lee SY: Microbial production of building block chemicals and polymers. Curr Opin Biotechnol 2011, 22:758-767.

5. Sauer M, Porro D, Mattanovich D, Branduardi P: Microbial production of organic acids: expanding the markets. Trends Biotechnol 2008, 26:100-108.

6. Falch EA: Industrial enzymes-developments in production and application. Biotechnol Adv 1991, 9:643-658.

7. Bouws $H$, Wattenberg A, Zorn H: Fungal secretomes-nature's toolbox for white biotechnology. Appl Microbiol Biotechnol 2008, 80:381-388.

8. Ferrer-Miralles N, Domingo-Espín J, Corchero J, Vázquez E, Villaverde A: Microbial factories for recombinant pharmaceuticals. Microb Cell Fact 2009, 8:17.

9. Ren Q, Ruth K, Thöny-Meyer L, Zinn M: Enatiomerically pure hydroxycarboxylic acids: current approaches and future perspectives. Appl Microbiol Biotechnol 2010, 87:41-52.

10. Lu T, Khalil A, Collins J: Next-generation synthetic gene networks. Nat Biotechnol 2009, 27:1139-1150.

11. Purnick $P$, Weiss $R$ : The second wave of synthetic biology: from modules to systems. Nat Rev Mol Cell Biol 2009, 10:410-422.

12. Kim T, Sohn S, Kim H, Lee S: Strategies for systems-level metabolic engineering. Biotechnol J 2008, 3:612-623.

13. Mukhopadhyay A, Redding A, Rutherford B, Keasling J: Importance of systems biology in engineering microbes for biofuel production. Curr Opin Biotechnol 2008, 19:228-234

14. Bro C, Nielsen J: Impact of 'ome' analyses on inverse metabolic engineering. Metab Eng 2004, 6:204-211.

15. Conrad TM, Lewis NE, Palsson B: Microbial laboratory evolution in the era of genome-scale science. Mol Syst Biol 2011, 7:509.

16. Portnoy VA, Bezdan D, Zengler K: Adaptive laboratory evolutionharnessing the power of biology for metabolic engineering. Curr Opin Biotechnol 2011, 22:590-594.

17. Nam H, Conrad TM, Lewis NE: The role of cellular objectives and selective pressures in metabolic pathway evolution. Curr Opin Biotechnol 2011, 22:595-600.

18. Oud B, Van Maris AJ, Daran JM, Pronk JT: Genome-wide analytical approaches for reverse metabolic engineering of industrially relevant phenotypes in yeast. FEMS Yeast Res 2012, 12:183-196.

19. Cakar ZP, Turanli-Yildiz B, Alkim C, Yilmaz U: Evolutionary engineering of Saccharomyces cerevisiae for improved industrially important properties. FEMS Yeast Res 2012, 12:171-182.

20. Dettman JR, Rodrigue N, Melnyk AH, Wong A, Bailey SF, Kassen R: Evolutionary insight from whole-genome sequencing of experimentally evolved microbes. Mol Ecol 2012, 21:2058-2077.

21. Mozhayskiy $V$, Tagkopoulos I: Microbial evolution in vivo and in silico: methods and applications. Integr Biol (Camb) 2013, 5:262-277.

22. Bennett AF, Hughes BS: Microbial experimental evolution. Am J Physiol Regul Integr Comp Physiol 2009, 297:R17-25.

23. Ryall B, Eydallin G, Ferenci T: Culture history and population heterogeneity as determinants of bacterial adaptation: the adaptomics of a single environmental transition. Microbiol Mol Biol Rev 2012, 76:597-625.

24. Silver $\mathrm{R}$, Mateles $\mathrm{R}$ : Control of mixed-substrate utilization in continuous cultures of Escherichia coli. J Bacterio/ 1969, 97:535-543. 
25. Lederberg J: Prevalence of Escherichia coli strains exhibiting genetic recombination. Science 1951, 114:68-69.

26. Bennett AF, Dao KM, Lenski RE: Rapid evolution in response to hightemperature selection. Nature 1990, 346:79-81.

27. Paquin $C$, Adams J: Frequency of fixation of adaptive mutations is higher in evolving diploid than haploid yeast populations. Nature 1983, 302:495-500.

28. Paquin CE, Adams J: Relative fitness can decrease in evolving asexual populations of S. cerevisiae. Nature 1983, 306:368-370.

29. Hardison RC: Comparative genomics. PLOS Biol 2003, 1:E58.

30. Schena M, Shalon D, Davis R, Brown P: Quantitative monitoring of gene expression patterns with a complementary DNA microarray. Science 1995, 270:467-470.

31. Liu L, Li Y, Li S, Hu N, He Y, Pong R, Lin D, Lu L, Law M: Comparison of next-generation sequencing systems. J Biomed Biotechnol 2012, 2012:251364.

32. Ansorge W: Next-generation DNA sequencing techniques. N Biotechnol 2009, 25:195-203.

33. Sniegowski P, Gerrish P, Lenski R: Evolution of high mutation rates in experimental populations of E. coli. Nature 1997, 387:703-705.

34. Lenski R, Mongold J, Sniegowski P, Travisano M, Vasi F, Gerrish P, Schmidt T: Evolution of competitive fitness in experimental populations of E. coli: what makes one genotype a better competitor than another? Antonie Van Leeuwenhoek 1998, 73:35-47.

35. Cooper V, Lenski R: The population genetics of ecological specialization in evolving Escherichia coli populations. Nature 2000, 407:736-739.

36. Barrick J, Yu D, Yoon S, Jeong H, Oh T, Schneider D, Lenski R, Kim J: Genome evolution and adaptation in a long-term experiment with Escherichia coli. Nature 2009, 461:1243-1247.

37. Blount Z, Borland C, Lenski R: Historical contingency and the evolution of a key innovation in an experimental population of Escherichia coli. Proc Natl Acad Sci USA 2008, 105:7899-7906.

38. Woods RJ, Barrick JE, Cooper TF, Shrestha U, Kauth MR, Lenski RE: Secondorder selection for evolvability in a large Escherichia coli population. Science 2011, 331:1433-1436.

39. Bloom J, Lu Z, Chen D, Raval A, Venturelli O, Arnold F: Evolution favors protein mutational robustness in sufficiently large populations. BMC Biol 2007, 5:29.

40. Elena SF, Wilke CO, Ofria C, Lenski RE: Effects of population size and mutation rate on the evolution of mutational robustness. Evolution 2007, 61:666-674.

41. Draghi J, Parsons T, Wagner G, Plotkin J: Mutational robustness can facilitate adaptation. Nature 2010, 463:353-355.

42. Kao KC, Sherlock G: Molecular characterization of clonal interference during adaptive evolution in asexual populations of Saccharomyces cerevisiae. Nat Genet 2008, 40:1499-1504.

43. Beaumont H, Gallie J, Kost C, Ferguson G, Rainey P: Experimental evolution of bet hedging. Nature 2009, 462:90-93.

44. Gonzalez A, Bell G: Evolutionary rescue and adaptation to abrupt environmental change depends upon the history of stress. Philos Trans $R$ Soc Lond B Biol Sci 2013, 368:20120079.

45. Wright J, Bellissimi E, De Hulster E, Wagner A, Pronk JT, Van Maris AJ: Batch and continuous culture-based selection strategies for acetic acid tolerance in xylose-fermenting Saccharomyces cerevisiae. FEMS Yeast Res 2011, 11:299-306.

46. Wang L, Spira B, Zhou Z, Feng L, Maharjan RP, Li X, Li F, McKenzie C, Reeves $P R$, Ferenci $T$ : Divergence involving global regulatory gene mutations in an Escherichia coli population evolving under phosphate limitation. Genome Biol Evol 2010, 2:478-487.

47. Koppram R, Albers E, Olsson L: Evolutionary engineering strategies to enhance tolerance of xylose utilizing recombinant yeast to inhibitors derived from spruce biomass. Biotechnol Biofuels 2012, 5:32.

48. Manch K, Notley-McRobb L, Ferenci T: Mutational adaptation of Escherichia coli to glucose limitation involves distinct evolutionary pathways in aerobic and oxygen-limited environments. Genetics 1999, 153:5-12.

49. Notley-McRobb L, Ferenci T: The generation of multiple co-existing malregulatory mutations through polygenic evolution in glucose-limited populations of Escherichia coli. Environ Microbiol 1999, 1:45-52.

50. Weikert C, Sauer U, Bailey J: Use of a glycerol-limited, long-term chemostat for isolation of Escherichia coli mutants with improved physiological properties. Microbiology 1997, 143(Pt 5):1567-1574.
51. Larsson G, Enfors SO, Pham H: The pH-auxostat as a tool for studying microbial dynamics in continuous fermentation. Biotechnol Bioeng 1990, 36:224-232

52. Zambrano MM, Siegele DA, Almirón M, Tormo A, Kolter R: Microbial competition: Escherichia coli mutants that take over stationary phase cultures. Science 1993, 259:1757-1760.

53. Wenger JW, Piotrowski J, Nagarajan S, Chiotti K, Sherlock G, Rosenzweig F: Hunger artists: yeast adapted to carbon limitation show trade-offs under carbon sufficiency. PLoS Genet 2011, 7:e1002202.

54. Rancati G, Pavelka N, Fleharty B, Noll A, Trimble R, Walton K, Perera A, Staehling-Hampton K, Seidel CW, Li R: Aneuploidy underlies rapid adaptive evolution of yeast cells deprived of a conserved cytokinesis motor. Cell 2008, 135:879-893.

55. Charusanti P, Fong NL, Nagarajan H, Pereira AR, Li HJ, Abate EA, Su Y, Gerwick WH, Palsson BO: Exploiting adaptive laboratory evolution of Streptomyces clavuligerus for antibiotic discovery and overproduction. PLoS One 2012, 7:e33727.

56. Feist AM, Zielinski DC, Orth JD, Schellenberger J, Herrgard MJ, Palsson B: Model-driven evaluation of the production potential for growth-coupled products of Escherichia coli. Metab Eng 2010, 12:173-186.

57. Schmidt FR: Optimization and scale up of industrial fermentation processes. Appl Microbiol Biotechnol 2005, 68:425-435.

58. Hahn-Hägerdal B, Karhumaa K, Larsson CU, Gorwa-Grauslund M, Görgens J Van Zyl WH: Role of cultivation media in the development of yeast strains for large scale industrial use. Microb Cell Fact 2005, 4:31.

59. Hughes B, Cullum A, Bennett A: Evolutionary adaptation to environmental pH in experimental lineages of Escherichia coli. Evolution 2007, 61:1725-1734.

60. Rainey PB, Beaumont HJ, Ferguson GC, Gallie J, Kost C, Libby E, Zhang XX: The evolutionary emergence of stochastic phenotype switching in bacteria. Microb Cell Fact 2011, 10:1-14.

61. Barton NH: Genetic hitchhiking. Philos Trans R Soc Lond B Biol Sci 2000, 355:1553-1562.

62. Cooper T, Lenski R: Experimental evolution with E. coli in diverse resource environments. I. Fluctuating environments promote divergence of replicate populations. BMC Evol Biol 2010, 10:11.

63. Dragosits M, Mozhayskiy V, Quinones-Soto S, Park J, Tagkopoulos I: Evolutionary potential, cross-stress behavior and the genetic basis of acquired stress resistance in Escherichia coli. Mol Syst Biol 2013, 9:643.

64. Cooper T, Rozen D, Lenski R: Parallel changes in gene expression after 20,000 generations of evolution in Escherichiacoli. Proc Natl Acad Sci USA 2003, 100:1072-1077

65. Ibarra R, Edwards J, Palsson B: Escherichia coli K-12 undergoes adaptive evolution to achieve in silico predicted optimal growth. Nature 2002, 420:186-189.

66. Fong S, Joyce A, Palsson B: Parallel adaptive evolution cultures of Escherichia coli lead to convergent growth phenotypes with different gene expression states. Genome Res 2005, 15:1365-1372.

67. Hua Q, Joyce AR, Palsson B, Fong SS: Metabolic characterization of Escherichia coli strains adapted to growth on lactate. Appl Environ Microbiol 2007, 73:4639-4647.

68. Dekel $\mathrm{E}$, Alon U: Optimality and evolutionary tuning of the expression level of a protein. Nature 2005, 436:588-592.

69. Conrad TM, Frazier M, Joyce AR, Cho BK, Knight EM, Lewis NE, Landick R, Palsson B: RNA polymerase mutants found through adaptive evolution reprogram Escherichia coli for optimal growth in minimal media. Proc Natl Acad Sci USA 2010, 107:20500-20505.

70. Charusanti P, Conrad TM, Knight EM, Venkataraman K, Fong NL, Xie B, Gao Y, Palsson B: Genetic basis of growth adaptation of Escherichia coli after deletion of pgi, a major metabolic gene. PLoS Genet 2010, 6:e1001186

71. Lee DH, Palsson B: Adaptive evolution of Escherichia coli K-12 MG1655 during growth on a Nonnative carbon source, L-1,2-propanediol. Appl Environ Microbiol 2010, 76:4158-4168.

72. Deng Y, Fong SS: Laboratory evolution and multi-platform genome resequencing of the cellulolytic actinobacterium Thermobifida fusca. J Biol Chem 2011, 286:39958-39966.

73. Bachmann H, Starrenburg MJ, Molenaar D, van Hylckama Vlieg JE, Kleerebezem M: Microbial domestication signatures of Lactococcus lactis can be reproduced by experimental evolution. Genome Res 2012, 22:115-124 
74. Summers ZM, Ueki T, Ismail W, Haveman SA, Lovley DR: Laboratory evolution of Geobacter sulfurreducens for enhanced growth on lactate via a single-base-pair substitution in a transcriptional regulator. ISME J 2012, 6:975-983.

75. Jiang L, Li S, Hu Y, Xu Q, Huang H: Adaptive evolution for fast growth on glucose and the effects on the regulation of glucose transport system in Clostridium tyrobutyricum. Biotechnol Bioeng 2012, 109:708-718.

76. Quan S, Ray JC, Kwota Z, Duong T, Balázsi G, Cooper TF, Monds RD: Adaptive evolution of the lactose utilization network in experimentally evolved populations of Escherichia coli. PLOS Genet 2012, 8:e1002444.

77. Zhao J, Xu L, Wang Y, Zhao X, Wang J, Garza E, Manow R, Zhou S: Homofermentative production of optically pure L-lactic acid from xylose by genetically engineered Escherichia coli B. Microb Cell Fact 2013, 12:57.

78. Riehle M, Bennett A, Lenski R, Long A: Evolutionary changes in heatinducible gene expression in lines of Escherichia coli adapted to high temperature. Physiol Genomics 2003, 14:47-58.

79. Alcántara-Díaz D, Breña-Valle M, Serment-Guerrero J: Divergent adaptation of Escherichia coli to cyclic ultraviolet light exposures. Mutagenesis 2004, 19:349-354.

80. Sleight S, Lenski R: Evolutionary adaptation to freeze-thaw-growth cycles in Escherichia coli. Physiol Biochem Zool 2007, 80:370-385.

81. Sleight S, Orlic C, Schneider D, Lenski R: Genetic basis of evolutionary adaptation by Escherichia coli to stressful cycles of freezing, thawing and growth. Genetics 2008, 180:431-443.

82. Stoebel D, Hokamp K, Last M, Dorman C: Compensatory evolution of gene regulation in response to stress by Escherichia coli lacking RpoS. PLoS Genet 2009, 5:e1000671.

83. Rudolph B, Gebendorfer KM, Buchner J, Winter J: Evolution of Escherichia coli for growth at high temperatures. J Biol Chem 2010, 285:19029-19034.

84. Goodarzi H, Bennett B, Amini S, Reaves M, Hottes A, Rabinowitz J: Tavazoie $\mathrm{S}$ : Regulatory and metabolic rewiring during laboratory evolution of ethanol tolerance in E. coli. Mol Syst Biol 2010, 6:378.

85. Horinouchi T, Tamaoka K, Furusawa C, Ono N, Suzuki S, Hirasawa T, Yomo T, Shimizu H: Transcriptome analysis of parallel-evolved Escherichia coli strains under ethanol stress. BMC Genomics 2010, 11:579.

86. Atsumi S, Wu TY, Machado IM, Huang WC, Chen PY, Pellegrini M, Liao JC: Evolution, genomic analysis, and reconstruction of isobutanol tolerance in Escherichia coli. Mol Syst Biol 2010, 6:449.

87. Minty JJ, Lesnefsky AA, Lin F, Chen Y, Zaroff TA, Veloso AB, Xie B, McConnell CA, Ward RJ, Schwartz DR, et al: Evolution combined with genomic study elucidates genetic bases of isobutanol tolerance in Escherichia coli. Microb Cell Fact 2011, 10:18.

88. Sen M, Yılmaz U, Baysal A, Akman S, Cakar ZP: In vivo evolutionary engineering of a boron-resistant bacterium: Bacillus boroniphilus. Antonie Van Leeuwenhoek 2011, 99:825-835.

89. Tenaillon $\mathrm{O}$, Rodríguez-Verdugo A, Gaut RL, McDonald P, Bennett AF, Long $A D$, Gaut BS: The molecular diversity of adaptive convergence. Science 2012, 335:457-461

90. Blaby IK, Lyons BJ, Wroclawska-Hughes E, Phillips GC, Pyle TP, Chamberlin SG, Benner SA, Lyons TJ, Crécy-Lagard V, Crécy E: Experimental evolution of a facultative thermophile from a mesophilic ancestor. Appl Environ Microbiol 2012, 78:144-155.

91. Reyes LH, Almario MP, Winkler J, Orozco MM, Kao KC: Visualizing evolution in real time to determine the molecular mechanisms of $n$-butanol tolerance in Escherichia coli. Metab Eng 2012, 14:579-590.

92. Lu L, Wei L, Zhu K, Wei D, Hua Q: Combining metabolic engineering and adaptive evolution to enhance the production of dihydroxyacetone from glycerol by Gluconobacter oxydans in a low-cost way. Bioresour Technol 2012, 117:317-324

93. Ferea $T$, Botstein $D$, Brown $P$, Rosenzweig R: Systematic changes in gene expression patterns following adaptive evolution in yeast. Proc Natl Acad Sci USA 1999, 96:9721-9726.

94. Dunham MJ, Badrane H, Ferea T, Adams J, Brown PO, Rosenzweig F, Botstein D: Characteristic genome rearrangements in experimental evolution of Saccharomyces cerevisiae. Proc Natl Acad Sci USA 2002, 99:16144-16149.

95. Sonderegger M, Sauer U: Evolutionary engineering of Saccharomyces cerevisiae for anaerobic growth on xylose. Appl Environ Microbiol 2003 69:1990-1998

96. Jansen ML, Daran-Lapujade P, De Winde JH, Piper MD, Pronk JT: Prolonged maltose-limited cultivation of Saccharomyces cerevisiae selects for cells with improved maltose affinity and hypersensitivity. Appl Environ Microbiol 2004, 70:1956-1963.

97. Van Maris AJ, Geertman JM, Vermeulen A, Groothuizen MK, Winkler AA, Piper MD, Van Dijken JP, Pronk JT: Directed evolution of pyruvate decarboxylase-negative Saccharomyces cerevisiae, yielding a C2independent, glucose-tolerant, and pyruvate-hyperproducing yeast. Appl Environ Microbiol 2004, 70:159-166

98. Jansen ML, Diderich JA, Mashego M, Hassane A, De Winde JH, DaranLapujade P, Pronk JT: Prolonged selection in aerobic, glucose-limited chemostat cultures of Saccharomyces cerevisiae causes a partial loss of glycolytic capacity. Microbiology 2005, 151:1657-1669.

99. Kuyper M, Toirkens MJ, Diderich JA, Winkler AA, Van Dijken JP, Pronk JT: Evolutionary engineering of mixed-sugar utilization by a xylosefermenting Saccharomyces cerevisiae strain. FEMS Yeast Res 2005, 5:925-934

100. Wisselink HW: Toirkens MJ, del Rosario Franco Berriel M, Winkler AA, van Dijken JP, Pronk JT, van Maris AJ: Engineering of Saccharomyces cerevisiae for efficient anaerobic alcoholic fermentation of L-arabinose. Appl Environ Microbiol 2007, 73:4881-4891.

101. Wisselink HW, Cipollina C, Oud B, Crimi B, Heijnen JJ, Pronk JT, Van Maris AJ: Metabolome, transcriptome and metabolic flux analysis of arabinose fermentation by engineered Saccharomyces cerevisiae. Metab Eng 2010, 12:537-551.

102. Wisselink HW, Toirkens MJ, Wu Q, Pronk JT, Van Maris AJ: Novel evolutionary engineering approach for accelerated utilization of glucose, xylose, and arabinose mixtures by engineered Saccharomyces cerevisiae strains. Appl Environ Microbiol 2009, 75:907-914.

103. Araya $C L$, Payen $C$, Dunham MJ, Fields S: Whole-genome sequencing of a laboratory-evolved yeast strain. BMC Genomics 2010, 11:88

104. Garcia Sanchez R, Karhumaa K, Fonseca C, Sànchez Nogué V, Almeida JR, Larsson CU, Bengtsson O, Bettiga M, Hahn-Hägerdal B, Gorwa-Grauslund MF: Improved xylose and arabinose utilization by an industrial recombinant Saccharomyces cerevisiae strain using evolutionary engineering. Biotechnol Biofuels 2010, 3:13

105. Zelle RM, Trueheart J, Harrison JC, Pronk JT, Van Maris AJ: Phosphoenolpyruvate carboxykinase as the sole anaplerotic enzyme in Saccharomyces cerevisiae. Appl Environ Microbiol 2010, 76:5383-5389.

106. Hong KK, Vongsangnak W, Vemuri GN, Nielsen J: Unravelling evolutionary strategies of yeast for improving galactose utilization through integrated systems level analysis. Proc Natl Acad Sci USA 2011, 108:12179-12184

107. Zelle RM, Harrison JC, Pronk JT, Van Maris AJ: Anaplerotic role for cytosolic malic enzyme in engineered Saccharomyces cerevisiae strains. Appl Environ Microbiol 2011, 77:732-738.

108. Shen $Y$, Chen X, Peng B, Chen L, Hou J, Bao X: An efficient xylosefermenting recombinant Saccharomyces cerevisiae strain obtained through adaptive evolution and its global transcription profile. Appl Microbiol Biotechnol 2012, 96:1079-1091.

109. Zhou H, Cheng JS, Wang BL, Fink GR, Stephanopoulos G: Xylose isomerase overexpression along with engineering of the pentose phosphate pathway and evolutionary engineering enable rapid xylose utilization and ethanol production by Saccharomyces cerevisiae. Metab Eng 2012, 14:611-622

110. Scalcinati G, Otero JM, Van Vleet JR, Jeffries TW, Olsson L, Nielsen J: Evolutionary engineering of Saccharomyces cerevisiae for efficient aerobic xylose consumption. FEMS Yeast Res 2012, 12:582-597.

111. De Kok S, Nijkamp JF, Oud B, Roque FC, De Ridder D, Daran JM, Pronk JT, Van Maris AJ: Laboratory evolution of new lactate transporter genes in a jen $1 \Delta$ mutant of Saccharomyces cerevisiae and their identification as ADY2 alleles by whole-genome resequencing and transcriptome analysis. FEMS Yeast Res 2012, 12:359-374.

112. Almario MP, Reyes $L H$, Kao KC: Evolutionary engineering of Saccharomyces cerevisiae for enhanced tolerance to hydrolysates of lignocellulosic biomass. Biotechnol Bioeng 2013. epub April 2013.

113. Cakar ZP, Seker UO, Tamerler C, Sonderegger M, Sauer U: Evolutionary engineering of multiple-stress resistant Saccharomyces cerevisiae. FEMS Yeast Res 2005, 5:569-578.

114. Cakar ZP, Alkim C, Turanli B, Tokman N, Akman S, Sarikaya M, Tamerler C, Benbadis L, François JM: Isolation of cobalt hyper-resistant mutants of Saccharomyces cerevisiae by in vivo evolutionary engineering approach. J Biotechnol 2009, 143:130-138. 
115. Selmecki AM, Dulmage K, Cowen LE, Anderson JB, Berman J: Acquisition of aneuploidy provides increased fitness during the evolution of antifungal drug resistance. PLOS Genet 2009, 5:e1000705.

116. Dhar R, Sägesser R, Weikert C, Yuan J, Wagner A: Adaptation of Saccharomyces cerevisiae to saline stress through laboratory evolution. J Evol Biol 2011, 24:1135-1153.

117. Adamo GM, Brocca S, Passolunghi S, Salvato B, Lotti M: Laboratory evolution of copper tolerant yeast strains. Microb Cell Fact 2012, 11:1.

118. Gray JC, Goddard MR: Sex enhances adaptation by unlinking beneficial from detrimental mutations in experimental yeast populations. BMC Evol Biol 2012, 12:43

119. Avrahami-Moyal L, Engelberg D, Wenger JW, Sherlock G, Braun S: Turbidostat culture of Saccharomyces cerevisiae W303-1A under selective pressure elicited by ethanol selects for mutations in SSD1 and UTH1. FEMS Yeast Res 2012, 12:521-533.

120. Dhar R, Sägesser R, Weikert C, Wagner A: Yeast adapts to a changing stressful environment by evolving cross-protection and anticipatory gene regulation. Mol Biol Evol 2013, 30:573-588.

121. Cadière A, Aguera E, Caillé S, Ortiz-Julien A, Dequin S: Pilot-scale evaluation the enological traits of a novel, aromatic wine yeast strain obtained by adaptive evolution. Food Microbiol 2012, 32:332-337.

122. Cadière A, Ortiz-Julien A, Camarasa C, Dequin S: Evolutionary engineered Saccharomyces cerevisiae wine yeast strains with increased in vivo flux through the pentose phosphate pathway. Metab Eng 2011, 13:263-271.

123. Tremblay PL, Summers ZM, Glaven RH, Nevin KP, Zengler K, Barrett CL, Qiu Y, Palsson BO, Lovley DR: A c-type cytochrome and a transcriptional regulator responsible for enhanced extracellular electron transfer in Geobacter sulfurreducens revealed by adaptive evolution. Environ Microbiol 2011, 13:13-23.

124. Day A, Schneider C, Schneider BL: Yeast cell synchronization. Methods Mol Biol 2004, 241:55-76

125. Aldea M, Herrero E, Esteve MI, Guerrero R: Surface density of major outer membrane proteins in Salmonella typhimurium in different growth conditions. J Gen Microbiol 1980, 120:355-367.

126. Lee $\mathrm{DH}$, Feist $\mathrm{AM}$, Barrett $\mathrm{CL}$, Palsson B: Cumulative number of cell divisions as a meaningful timescale for adaptive laboratory evolution of Escherichia coli. PLoS One 2011, 6:e26172.

127. Drake JW: A constant rate of spontaneous mutation in DNA-based microbes. Proc Natl Acad Sci USA 1991, 88:7160-7164.

128. Adamo GM, Lotti M, Tamás MJ, Brocca S: Amplification of the CUP1 gene is associated with evolution of copper tolerance in Saccharomyces cerevisiae. Microbiology 2012, 158:2325-2335.

129. Innan H, Kondrashov F: The evolution of gene duplications: classifying and distinguishing between models. Nat Rev Genet 2010, 11:97-108.

130. Kondrashov FA, Kondrashov AS: Role of selection in fixation of gene duplications. J Theor Biol 2006, 239:141-151.

131. Bergthorsson U, Andersson DI, Roth JR: Ohno's dilemma: evolution of new genes under continuous selection. Proc Natl Acad Sci USA 2007, 104:17004-17009.

132. Maslov S, Krishna S, Pang TY, Sneppen K: Toolbox model of evolution of prokaryotic metabolic networks and their regulation. Proc Natl Acad Sci USA 2009, 106:9743-9748.

133. Boto L: Horizontal gene transfer in evolution: facts and challenges. Proc Biol Sci 2010, 277:819-827.

134. Pál C, Papp B, Lercher MJ, Csermely P, Oliver SG, Hurst LD: Chance and necessity in the evolution of minimal metabolic networks. Nature 2006, 440:667-670.

135. Takemoto K, Nacher JC, Akutsu T: Correlation between structure and temperature in prokaryotic metabolic networks. BMC Bioinforma 2007, 8:303.

136. Whitacre J, Bender A: Degeneracy: A design principle for achieving robustness and evolvability. J Theor Biol 2010, 263:143-153.

137. Stephanopoulos GN, Aristidou AA, Nielsen J: Metabolic Engineering. Principles and methologies. San Diego. USA: Academic Press; 1998. ISBN ISBN 0-12 $-666260-6$.

138. Nielsen J: Metabolic engineering: techniques for analysis of targets for genetic manipulations. Biotechnol Bioeng 1998, 58:125-132.

139. Giraud A, Matic I, Tenaillon O, Clara A, Radman M, Fons M, Taddei F: Costs and benefits of high mutation rates: adaptive evolution of bacteria in the mouse gut. Science 2001, 291:2606-2608.
140. Notley-McRobb L, Seeto S, Ferenci T: Enrichment and elimination of mutY mutators in Escherichia coli populations. Genetics 2002, 162:1055-1062.

141. Notley-McRobb L, Pinto R, Seeto S, Ferenci T: Regulation of mutY and nature of mutator mutations in Escherichia coli populations under nutrient limitation. J Bacterio/ 2002, 184:739-745.

142. Darlington PJ: The cost of evolution and the imprecision of adaptation. Proc Natl Acad Sci USA 1977, 74:1647-1651.

143. Wielgoss S, Barrick JE, Tenaillon O, Wiser MJ, Dittmar WJ, Cruveiller S, ChaneWoon-Ming B, Médigue C, Lenski RE, Schneider D: Mutation rate dynamics in a bacterial population reflect tension between adaptation and genetic load. Proc Natl Acad Sci USA 2013, 110:222-227.

144. Khersonsky O, Roodveldt C, Tawfik DS: Enzyme promiscuity: evolutionary and mechanistic aspects. Curr Opin Chem Biol 2006, 10:498-508.

145. Jarosz DF, Lindquist S: Hsp90 and environmental stress transform the adaptive value of natural genetic variation. Science 2010, 330:1820-1824

146. Cowen LE, Lindquist S: Hsp90 potentiates the rapid evolution of new traits: drug resistance in diverse fungi. Science 2005, 309:2185-2189.

147. Veeravalli K, Boyd D, Iverson BL, Beckwith J, Georgiou G: Laboratory evolution of glutathione biosynthesis reveals natural compensatory pathways. Nat Chem Biol 2011, 7:101-105

148. Finkel SE: Long-term survival during stationary phase: evolution and the GASP phenotype. Nat Rev Microbiol 2006, 4:113-120.

149. Zinser ER, Kolter R: Mutations enhancing amino acid catabolism confer a growth advantage in stationary phase. J Bacteriol 1999, 181:5800-5807.

150. Crozat E, Winkworth C, Gaffé J, Hallin PF, Riley MA, Lenski RE, Schneider D: Parallel genetic and phenotypic evolution of DNA superhelicity in experimental populations of Escherichia coli. Mol Biol Evol 2010, 27:2113-2128.

151. Oud B, Flores CL, Gancedo C, Zhang X, Trueheart J, Daran JM, Pronk JT, Van Maris AJ: An internal deletion in MTH1 enables growth on glucose of pyruvate-decarboxylase negative, non-fermentative Saccharomyces cerevisiae. Microb Cell Fact 2012, 11:131

152. Tang YC, Amon A: Gene copy-number alterations: a cost-benefit analysis. Cell 2013, 152:394-405

153. Pérez-Ortín JE, Querol A, Puig S, Barrio E: Molecular characterization of a chromosomal rearrangement involved in the adaptive evolution of yeast strains. Genome Res 2002, 12:1533-1539.

154. Hittinger CT, Carroll SB: Gene duplication and the adaptive evolution of a classic genetic switch. Nature 2007, 449:677-681.

155. Dujon B: Yeasts illustrate the molecular mechanisms of eukaryotic genome evolution. Trends Genet 2006, 22:375-387.

156. Dujon B, Sherman D, Fischer G, Durrens P, Casaregola S, Lafontaine I, De Montigny J, Marck C, Neuvéglise C, Talla E, et al: Genome evolution in yeasts. Nature 2004, 430:35-44

157. Pelosi L, Kühn L, Guetta D, Garin J, Geiselmann J, Lenski R, Schneider D: Parallel changes in global protein profiles during long-term experimental evolution in Escherichia coli. Genetics 2006, 173:1851-1869.

158. Ferenci T: Maintaining a healthy SPANC balance through regulatory and mutational adaptation. Mol Microbiol 2005, 57:1-8.

159. Philippe N, Crozat E, Lenski R, Schneider D: Evolution of global regulatory networks during a long-term experiment with Escherichia coli. BioEssays 2007, 29:846-860

160. Levy S, Ihmels J, Carmi M, Weinberger A, Friedlander G, Barkai N: Strategy of transcription regulation in the budding yeast. PLoS One 2007, 2:e250.

161. Brauer MJ, Huttenhower C, Airoldi EM, Rosenstein R, Matese JC, Gresham D, Boer VM, Troyanskaya OG, Botstein D: Coordination of growth rate, cell cycle, stress response, and metabolic activity in yeast. Mol Biol Cell 2008, 19:352-367.

162. Hong KK, Nielsen J: Adaptively evolved yeast mutants on galactose show trade-offs in carbon utilization on glucose. Metab Eng 2013, 16:78-86.

163. Bennett A, Lenski R: An experimental test of evolutionary trade-offs during temperature adaptation. Proc Natl Acad Sci USA 2007, 104(Suppl 1):8649-8654.

164. Blumer-Schuette SE, Kataeva I, Westpheling J, Adams MW, Kelly RM: Extremely thermophilic microorganisms for biomass conversion: status and prospects. Curr Opin Biotechnol 2008, 19:210-217.

165. Turner $P$, Mamo G, Karlsson EN: Potential and utilization of thermophiles and thermostable enzymes in biorefining. Microb Cell Fact 2007, 6:9.

166. Schiraldi C, Giuliano M, De Rosa M: Perspectives on biotechnological applications of archaea. Archaea 2002, 1:75-86. 
167. Corchero JL, Gasser B, Resina D, Smith W, Parrilli E, Vázquez F, Abasolo I, Giuliani $M$, Jäntti J, Ferrer $P$, et al: Unconventional microbial systems for the cost-efficient production of high-quality protein therapeutics. Biotechnol Adv 2013, 31:140-153.

168. Johnson EA: Biotechnology of non-Saccharomyces yeasts-the ascomycetes. Appl Microbiol Biotechnol 2013, 97:503-517.

169. Nikolaou E, Agrafioti I, Stumpf M, Quinn J, Stansfield I, Brown A: Phylogenetic diversity of stress signalling pathways in fungi. BMC Evol Biol 2009, 9:44

170. Gasch A: Comparative genomics of the environmental stress response in ascomycete fungi. Yeast 2007, 24:961-976.

171. Crombach A, Hogeweg P: Evolution of evolvability in gene regulatory networks. PLoS Comput Biol 2008, 4:e1000112.

172. Tagkopoulos I, Liu Y, Tavazoie S: Predictive behavior within microbial genetic networks. Science 2008, 320:1313-1317.

173. Mitchell A, Romano G, Groisman B, Yona A, Dekel E, Kupiec M, Dahan O, Pilpel Y: Adaptive prediction of environmental changes by microorganisms. Nature 2009, 460:220-224.

174. Liu M, Durfee T, Cabrera J, Zhao K, Jin D, Blattner F: Global transcriptional programs reveal a carbon source foraging strategy by Escherichia coli. J Biol Chem 2005, 280:15921-15927.

175. Thomson JM, Gaucher EA, Burgan MF, De Kee DW, Li T, Aris JP, Benner SA: Resurrecting ancestral alcohol dehydrogenases from yeast. Nat Genet 2005, 37:630-635.

176. Miyazaki T, Nakayama H, Nagayoshi Y, Kakeya H, Kohno S: Dissection of Ire1 Functions Reveals Stress Response Mechanisms Uniquely Evolved in Candida glabrata. PLoS Pathog 2013, 9:e1003160.

177. Vaupotic T, Plemenitas A: Differential gene expression and Hog1 interaction with osmoresponsive genes in the extremely halotolerant black yeast Hortaea werneckii. BMC Genomics 2007, 8:280.

178. Sauer U: Evolutionary engineering of industrially important microbial phenotypes. Adv Biochem Eng Biotechnol 2001, 73:129-169.

179. Mozhayskiy V, Tagkopoulos I: Guided evolution of in silico microbial populations in complex environments accelerates evolutionary rates through a step-wise adaptation. BMC Bioinforma 2012, 13:10

180. Wang HH, Isaacs FJ, Carr PA, Sun ZZ, Xu G, Forest CR, Church GM: Programming cells by multiplex genome engineering and accelerated evolution. Nature 2009, 460:894-898.

181. Isalan M, Lemerle C, Michalodimitrakis K, Horn C, Beltrao P, Raineri E, Garriga-Canut M, Serrano L: Evolvability and hierarchy in rewired bacterial gene networks. Nature 2008, 452:840-845.

182. Chen T, Wang J, Yang R, Li J, Lin M, Lin Z: Laboratory-evolved mutants of an exogenous global regulator, IrrE from Deinococcus radiodurans, enhance stress tolerances of Escherichia coli. PLoS One 2011, 6:e16228

183. Andrianantoandro E, Basu S, Karig DK, Weiss R: Synthetic biology: new engineering rules for an emerging discipline. Mol Syst Biol 2006 2:2006.0028

184. Porcar M: Beyond directed evolution: Darwinian selection as a tool for synthetic biology. Syst Synth Biol 2010, 4:1-6.

185. Karr JR, Sanghvi JC, Macklin DN, Gutschow MV, Jacobs JM, Bolival B, AssadGarcia N, Glass Jl, Covert MW: A whole-cell computational model predicts phenotype from genotype. Cell 2012, 150:389-401.

186. Orth JD, Conrad TM, Na J, Lerman JA, Nam H, Feist AM, Palsson B: A comprehensive genome-scale reconstruction of Escherichia coli metabolism-2011. Mol Syst Biol 2011, 7:535.

187. Wiechert W, Noack S: Mechanistic pathway modeling for industrial biotechnology: challenging but worthwhile. Curr Opin Biotechnol 2011, 22:604-610.

doi:10.1186/1475-2859-12-64

Cite this article as: Dragosits and Mattanovich: Adaptive laboratory evolution - principles and applications for biotechnology. Microbial Cell Factories 2013 12:64.

\section{Submit your next manuscript to BioMed Central and take full advantage of:}

- Convenient online submission

- Thorough peer review

- No space constraints or color figure charges

- Immediate publication on acceptance

- Inclusion in PubMed, CAS, Scopus and Google Scholar

- Research which is freely available for redistribution

Submit your manuscript at www.biomedcentral.com/submit
Biomed Central 